\title{
INTERVENÇÃO DE ENFERMAGEM AO PACIENTE EM CRISE PSIQUIÁTRICA NOS CENTROS DE ATENÇÃO PSICOSSOCIAL*
}

Heloísa Helena de Sousa e Silva ${ }^{1}$, Priscilla Maria de Castro Silva², Elisângela Braga de Azevedo ${ }^{3}$, Divanda Cruz Rocha ${ }^{4}$, Lorena de Farias Pimentel Costa ${ }^{5}$, Juliana de Oliveira Musse ${ }^{6}$

RESUMO: Estudo de caráter descritivo com abordagem qualitativa objetivou investigar estratégias de intervenção de enfermagem direcionadas ao usuário em crise psiquiátrica, identificando os fatores que as desencadeiam. Fizeram parte do estudo dois enfermeiros e 14 técnicos em enfermagem atuantes nos Centros de Atenção Psicossocial de Campina Grande, Paraíba. Os dados foram coletados entre abril e maio de 2011 por meio de entrevista semiestruturada e analisados por meio da técnica de análise de conteúdo. As estratégias referidas foram a escuta/conversa, uso de psicofármacos e contensão. Em relação aos fatores desencadeantes identificou-se razões de cunho familiar, uso irregular de medicação e abstinência de drogas. Pode-se perceber que as práticas de enfermagem estão baseadas no novo modelo de saúde dispensado em saúde mental.

PALAVRAS-CHAVE: Enfermagem; Serviços de saúde mental; Sofrimento psíquico.

\section{NURSING INTERVENTIONS FOR PATIENTS IN PSYCHIATRIC CRISIS IN PSYCHOSOCIAL CARE CENTERS}

\begin{abstract}
This descriptive study with a qualitative approach aimed to investigate strategies for nursing interventions directed at health service users in psychiatric crisis, identifying the factors which unleash this condition. Two nurses and 14 assistant nurses took part in the study, all working in Psycho-Social Care Centers in Campina Grande in the state of Paraíba, Brazil. The data was collected between April and May 2011, through semi-structured interviews, and was analyzed through the technique of content analysis. The strategies referred to were listening /conversing, use of psychopharmaceuticals, and containment. In relation to the factors which lead to the crisis, the following were identified: reasons related to the family, irregular use of medication and abstinence from drugs. It may be noted that the nursing practices are based in the new model of health dispensed in mental health.

KEYWORDS: Nursing; Mental health services; Psychiatric suffering.

\section{INTERVENCIÓN DE ENFERMERÍA AL PACIENTE EN CRISIS PSIQUIÁTRICO EN LOS CENTROS DE ATENCIÓN PSICOSOCIAL}

RESUMEN: Estudio de carácter descriptivo con abordaje cualitativo cuyo objetivo fue investigar estrategias de intervención de Enfermería para el usuario en crisis psiquiátrico, identificando los factores que las causan. Hicieron parte del estudio dos enfermeros y 14 técnicos en Enfermería actuantes en los Centros de Atención Psicosocial de Campina Grande, Paraíba. Los datos fueron obtenidos entre abril y mayo de 2011 por medio de entrevista semiestructurada y analizados utilizándose la técnica de análisis de contenido. Las estrategias fueron la escucha/charla, uso de psicofármacos y contensión. Los factores desencadenantes identificados fueron razones relacionadas a la familia, uso irregular de medicación y abstinencia de drogas. Se puede percibir que las prácticas de Enfermería están basadas en el nuevo modelo de salud usado en salud mental. PALABRAS CLAVES: Enfermería; Servicios de salud mental; Sufrimiento psíquico.

\footnotetext{
* Este artigo é um recorte do Trabalho de Conclusão de Curso da Graduação em Enfermagem da Faculdade de Ciências Médicas de Campina Grande - PB, intitulado: As práticas de Enfermagem nos Centros de Antenção Psicossocial de Campina Grande direcionadas ao paciente em crise psiquiátrica.

${ }^{1}$ Enfermeira.

${ }^{2}$ Enfermeira. Mestranda pelo Programa de Pós-Graduação em Enfermagem da Universidade Federal da Paraíba - UFPB. Professora do Departamento de Enfermagem da Universidade Federal de Campina Grande - PB. Membro do Grupo de Estudos em Saúde Mental Comunitária. ${ }^{3}$ Enfermeira. Doutoranda pelo Programa de Pós-Graduação em Enfermagem da UFPB. Professora do Departamento de Enfermagem da Universidade Estadual da Paraíba e da Faculdade de Ciências Médicas. Membro do Grupo de Estudos em Saúde Mental Comunitária. ${ }^{4}$ Enfermeira. Especialista em Pediatria e Puericultura e Enfermagem do Trabalho. Professora do Departamento de Enfermagem da Universidade Estadual da Paraíba e da Faculdade de Ciências Médicas. Membro do Grupo de Estudos em Saúde Mental Comunitária. ${ }^{5}$ Acadêmica de Enfermagem da Faculdade de Ciências Médicas de Campina Grande-PB. Membro do Grupo de Estudos em Saúde Mental Comunitária. ${ }^{6}$ Enfermeira. Mestranda em Saúde Pública pela Universidade Estadual da Paraíba.
}

Autor correspondente:

Juliana de Oliveira Musse

Universidade Estadual da Paraíba

R. Alvino de Farias Pimentel, 36 - 58411-010 -Campina Grande-PB-Brasil

E-mail: julimusse@hotmail.com
Recebido: 24/11/2011

Aprovado: 25/07/2012 


\section{INTRODUÇÃO}

Anteriormente no Brasil a assistência às pessoas em situação de sofrimento psíquico era realizada em hospitais psiquiátricos que, por sua vez, utilizavam métodos agressivos e violentos, como por exemplo, sessões de eletrochoque, uso de camisa de força, altas dosagens de medicamentos, dentre outros. Ao longo dos tempos, buscou-se formas de mudar essa realidade, emergindo, com isso, a Reforma Psiquiátrica ${ }^{(1)}$.

A Reforma Psiquiátrica no Brasil surgiu na década de 1980 com a necessidade de levar novas propostas e possibilidades de tratamento e cuidado ao paciente em situação de sofrimento psíquico. Com a reorganização do modelo de assistência psiquiátrica, emergiu um novo modelo extra-hospitalar, denominado de Centro de Atenção Psicossocial (CAPS) e, consequentemente, toda uma rede de cuidado em saúde mental foi construída progressivamente para receber usuários em situação de sofrimento psíquico ${ }^{(2)}$.

É nessa direção que os CAPS fazem parte de uma nova política de saúde e foram criados com o objetivo de implantar novas formas de cuidado aos portadores de sofrimento psíquico visando à redução do número de internações psiquiátrica, promovendo atividades que resgate o cliente para o espaço social. A implementação desse cuidado em saúde mental vem ocorrendo de forma gradual, mesmo havendo a necessidade da aceleração desse processo ${ }^{(2)}$.

Tais serviços oferecem atendimento diário, individualizado e acompanhamento clínico a partir de profissionais capacitados. São oferecidas atividades terapêuticas de diversos tipos como psicoterapia individual ou em grupo, orientação e acompanhamento de medicações, oficinas terapêuticas, atividades comunitárias, além de visita domiciliares e intersetoriais ${ }^{(2)}$.

A consulta de enfermagem à pessoa em situação de sofrimento psíquico deve ser embasada no novo modelo de cuidado, com o objetivo de renovar o atendimento e possibilitar ao usuário uma nova formar de viver ${ }^{(1)}$. Esta intervenção deve apoiar-se em atividades que possibilitem a convivência, a interação e que promovam a sua inserção na sociedade ${ }^{(3)}$ voltada para sua autonomia e melhoria da qualidade de vida.

Por mais qualificado que seja o cuidado prestado pelos profissionais do CAPS ao portador de sofrimento psíquico existem fatores intrínsecos e extrínsecos que possibilitam o desenvolvimento de um período de crise. Com esse entendimento, revela-se que, no meio científico, a causa definitiva para o desenvolvimento dos transtornos mentais, ainda não foi comprovada. Porém, sua relação com fatores hereditários e traumas psíquicos no período de infância e, posteriormente, são considerados grandes aliados para o desenvolvimento de $\operatorname{crises}^{(4)}$.

Frequentemente, todos passam por situações de conflito e desequilíbrio durante a vida, podem acarretar um período de crise que, posteriormente será facilmente superado. Para aqueles que apresentam crises do tipo psicóticas, que aparentemente surgem de forma abrupta, carregam consigo alguns traços de alteração no estado emocional que indicam um momento de surto posterior, são exemplos: isolamento, comportamento estranho ou agressivo, discurso confuso, descuido para com a higienização e possíveis alucinações ${ }^{(5)}$.

Quando o paciente apresenta uma crise psiquiátrica o seu funcionamento geral é prejudicado, ocorre um período de desequilíbrio psíquico, e assim, o usuário encontra-se desprovido de competências, dificultando, desse modo, que este assuma suas responsabilidades pessoais. Nesse contexto, o acompanhamento de enfermagem torna-se ainda mais essencial para a mobilização de recursos necessários na resolução da crise ${ }^{(6)}$.

O enfermeiro deve possuir controle emocional e habilidades técnicas para identificar quais foram os fatores desencadeadores ${ }^{(7)}$. No entanto, muitas vezes a equipe de enfermagem não se encontra familiarizada com alguns aspectos que envolvem a intervenção na crise psiquiátrica ${ }^{()}$.

Para tanto, pergunta-se: Quais são as estratégias de intervenção da enfermagem com os usuários em situação de crise psiquiátrica nos Centros de Atenção Psicossocial? Quais os fatores que tem desencadeado as crises psiquiátricas nos usuários assistidos nos Centros de Atenção Psicossocial?

Nesta perspectiva, este estudo objetivou investigar as estratégias de intervenção da Enfermagem direcionadas ao usuário em crise psiquiátrica, quando os mesmos se encontram nos Centros de Atenção Psicossocial, identificando os fatores que desencadeiam as crises psiquiátricas.

\section{MÉTODO}

Trata-se de um estudo descritivo, com abordagem qualitativa. A pesquisa foi realizada nos CAPS do Município de Campina Grande, Paraíba, no período de abril a maio de 2011. Participaram do estudo dois enfermeiros e 14 técnicos em enfermagem, com os seguintes critérios de inclusão: 1) ser atuante em CAPS do Município de Campina Grande, 2) ter vinculo empregatício com o município há pelo menos um ano.

Como instrumento para coleta do material empírico 
utilizou-se a entrevista semiestruturada. As entrevistas foram gravadas em um aparelho de Mídia Player (MP3), tendo sido realizada após comunicar aos mesmos sobre os objetivos, justificativas e procedimentos utilizados na investigação.

O material empírico foi analisado por meio da técnica de análise de conteúdo ${ }^{(8)}$, que tem como objetivo realizar a categorização das informações por homogeneidade, exaustividade, exclusividade, objetividade e adequação ou pertinência. Como fase para a análise de conteúdo foi realizada a transcrição das entrevistas em sua literalidade, categorizando-as de acordo com suas afinidades e pertinência. Com o intuito de manter o sigilo dos entrevistados, foi utilizado códigos para identificá-los.

Para a apresentação do material empírico, utilizou-se a técnica da narrativa que possibilitou o confronto de significados com a literatura pertinente ao tema discutido. Foram apresentados em forma de categorias temáticas, formadas de acordo com o surgimento do eixo temático e/ou palavras nas falas dos entrevistados.

O estudo foi desenvolvido de acordo com os aspectos éticos da pesquisa envolvendo seres humanos recomendados pela resolução 196/96 do Conselho Nacional de Saúde. A pesquisa foi submetida à apreciação do Comitê de Ética em Pesquisa do Centro de Ensino Superior e Desenvolvimento - CESED, que emitiu parecer favorável em 28/04/2011, segundo protocolo CEP/CAAE -0005.0.405.000-11.

\section{RESULTADOS}

Tendo em vista a importância das estratégias utilizadas nas intervenções de enfermagem com o paciente em crise, foram agrupadas as seguintes categorias temáticas, descritas abaixo, com as respectivas subcategorias.

\section{Estratégias para intervenção do paciente em crise}

Nesta categoria foram desdobradas as estratégias utilizadas nas intervenções de enfermagem com o paciente em crise, em duas subcategorias:

\section{Escuta/Conversa}

A escuta e a conversa foi apontada pelos profissionais como estratégias de restabelecimento do portador de sofrimento psíquico:
A escuta é a primeira estratégia usada com o paciente. (E3)

Dependendo da intensidade da crise, pode ser realizada uma conversa na tentativa de acamá-lo [...]. (E14)

\section{Medicação/Contenção}

O uso de psicofármacos e da contenção no tratamento do portador de sofrimento psíquico também se faz necessário, segundo os participantes:

[...] infelizmente a gente ainda utiliza da medicação e da contenção $[\ldots .].(\mathrm{E} 4)$

Em último caso contê-lo, fazer medicação injetável [...] (E10)

\section{Desencadeamento das crises}

Entre as causas que desencadeiam as crises, os profissionais consideram fatores familiares, medicamentosos e de abstinência, apresenta-se abaixo as subcategorias.

\section{Razões intrafamiliares}

A família é apontada como o centro de apoio dos usuários em sofrimento mental, possui uma forte repercussão sobre as crises psíquicas, como pôde ser observado:

[...] a família é um centro de apoio muito grande, eles precisam realmente desse apoio familiar, se não tiver desencadeia a crise [...] (E7)

Muitas vezes falta de apoio da família [...] (E11)

\section{Uso irregular da medicação}

O uso irregular da medicação, segundo os profissionais, é um dos fatores que levam o paciente com sofrimento psíquico a ter recaídas e momentos de crise:

[...] pode ocorrer devido a falta do uso da medicação ou até mesmo da automedicação. (E2)

Algumas vezes o usuário faz uso incorreto da medicação [...] (E3)

[...] às vezes o uso irregular de medicação. (E8) 


\section{Abstinência das drogas}

Faz-se necessário que os pacientes estejam adaptados a abstinência, para que estes possam atravessar o tratamento sem recaídas ou crises. Essa adaptação é importante, pois irá contribuir para seu tratamento.

Mesmo em acompanhamento alguns usuários chegam a ter recaídas e ficam em abstinência [...] (E9)

\section{Referência e contrarreferência dos casos de crise}

Os profissionais referiram recorrer ao sistema de referência e contrarreferência quando o usuário está em crise. Emergiram da categoria supracitada as subcategorias:

\section{Emergência Psiquiátrica}

Diante de crises psiquiátricas graves, os sujeitos da pesquisa relataram encaminhar os pacientes para a emergência psiquiátrica:

O usuário no momento inicial é encaminhado para a emergência psiquiátrica [...]. (E1)

[...] quando no caso mais grave é encaminhado para a emergência psiquiátrica. (E2)

\section{Hospital Psiquiátrico}

O hospital psiquiátrico ainda constitui uma referência diante de algumas crises psicóticas:

[...] diante de tal crise, poderá ser encaminhado ao hospital psiquiátrico. (E12)

\section{[...] conforme o caso, hospital psiquiátrico. (E13)}

\section{Segurança no manejo da crise}

Quando indagados em relação à segurança no manejo da crise, os profissionais referiram:

Até o momento, me sinto segura [...] (E1)

Depende de cada caso e de como o usuário se encontre [...]. (E2)

\section{Emoções ao lidar com a crise}

Esses achados referem-se às emoções do profissional de enfermagem ao lidar com o paciente em crise. De acordo com os depoimentos abaixo, sentimentos de medo, insegurança se misturam durante o manejo da crise.

Medo, porém não deixamos de dar assistência [...]. (E2)

Tranquila em algumas situações e insegura diante do paciente agressivo na crise. (E3)

Insegura, muitas vezes a gente fica com receio de ser agredida por eles [...]. (E6)

Algumas vezes inútil e também um pouco abalada [...]. (E15)

\section{Capacitação e/ou treinamento para manejar o pa- ciente em crise}

Esta categoria aborda se o profissional de enfermagem recebeu capacitação e/ou treinamento para manejar o paciente em crise, e de como isso influência na assistência direcionada ao paciente em crise. Emergiram as subcategorias:

\section{Não houve capacitação elou treinamento}

Alguns profissionais referiram não receber capacitação voltada para lidar com emergências psiquiátricas:

Capacitação para manejar o usuário em crise? não tive [.. .]. (E1)

A gente nunca teve capacitação direcionada para a enfermagem não! [...] (E8)

Não, nunca tivemos capacitação para lidar com esse problema. (E15)

Até o momento não. (E3)

Capacitação elou treinamento na implantação dos Serviços Substitutivos

É importante que ao manejar o paciente em crise, o profissional de enfermagem saiba o que está fazendo, para não colocar em risco sua segurança ou a do usuário. Alguns profissionais relataram receber o curso de capacitação: 
[...] teve uma capacitação sim. (E5)

Nós temos a capacitação [...]. (E6)

Houve no início quando começou a saúde mental [...]. (E7)

\section{DISCUSSÃO}

Entre as estratégias utilizadas pelos profissionais para intervenção do paciente em crise foram destacadas a escuta e conversa, o uso de medicamentos e contenção. A escuta e a conversa são de fundamental importância pois contribuem para o restabelecimento do portador de sofrimento psíquico. Dessa forma, a equipe de enfermagem pode interagir com o usuário, procurando saber o que está acontecendo, e dessa forma, tentar acalmá-lo.

A escuta e a comunicação entre profissional e paciente é necessária para que o profissional de enfermagem reconheça as necessidades do portador de sofrimento psíquico. Por isso, a comunicação passa a ser uma alternativa de intervenção e contribui para um bom resultado no tratamento ${ }^{(9)}$.

Também foi citado pelos entrevistados o uso de psicofármacos no tratamento do portador de sofrimento psíquico, que é de muita relevância, pois evita que o paciente tenha recaídas, provocando uma internação desnecessária. Este tipo de terapêutica modificou a vida dos pacientes com sofrimento psíquico, pois possibilita a realização de atividades práticas da vida. A utilização desses medicamentos auxilia na estabilização do paciente e facilita a convivência com a família ${ }^{(10)}$.

Dentre os motivos que desencadeiam as crises psiquiátricas foram evidenciadas razões intrafamiliares, o uso irregular das medicações e a abstinência de drogas. Como exemplos de atitudes que fazem parte da rotina de familiares de portadores de sofrimento psíquico, destacam-se as atitudes de carinho, cuidado e zelo, assim como proteção, afeto e compreensão. Este tipo de relacionamento afetivo familiar saudável, proporciona bem-estar e qualidade de tratamento ${ }^{(11)}$. Desse modo, entende-se que a família é uma das instituições responsáveis em cuidar do usuário com sofrimento psíquico e, por conseguinte, ela tornou-se o centro de apoio. Mas, quando ela deixa de ser esse centro de apoio, pode contribuir para o desencadeamento das crises. Apesar da inclusão da família no tratamento do usuário, alguns familiares não tem estrutura necessária para a superação e execução dos objetivos do tratamento. O convívio familiar, o despreparo da família em lidar com o usuário, a rejeição e o sentimento de inutilidade, tudo isso, contribui para que o usuário entre em crise psiquiátrica ${ }^{(12)}$.

Observando outra vertente de possibilidade de desencadeamento das crises, o profissional depara-se também com o uso irregular da medicação, que por outro lado, é um dos principais fatores que levam o usuário a ter recaídas, momentos de crise e a uma possível internação no hospital psiquiátrico, além de trazer vários danos e agravar o quadro da doença. É importante que os profissionais de enfermagem reconheçam o motivo pelo qual o usuário não está fazendo o uso correto da medicação e, a partir daí, elabore-se o projeto terapêutico individual, para que esse usuário volte a fazer uso correto da medicação, e com esta atitude, evitar novas crises ${ }^{(10)}$.

A abstinência das drogas interfere no tratamento, tendo em vista a severidade de algumas drogas, ou porque o usuário não tem consciência do problema, ou ainda, em decorrência da depressão que é desencadeada devido a abstinência ${ }^{(12)}$. Para que o cuidado a pessoa em situação de sofrimento psíquico seja de boa qualidade é necessário que o profissional de enfermagem tenha resolubilidade no serviço. Porém, quando tal fato não é possível, é preciso que esse encaminhe o usuário para outro serviço de saúde. Dentre os órgãos que os profissionais referiram recorrer, encontram-se as emergências psiquiátricas e aos hospitais psiquiátricos.

Os serviços de referência e contrarreferência funcionam como um sistema que garante o acesso livre, geral e apropriado dos pacientes no serviço, adequando-se a seu problema ${ }^{(13)}$. A emergência psiquiátrica é um serviço de saúde que surge como apoio aos CAPS, tem como objetivo reduzir o tempo de internação do paciente. Contudo, é função também dos profissionais que atuam na emergência, buscar a ressocialização do paciente enquanto este permanecer no serviço ${ }^{(14)}$. Quanto ao hospital psiquiátrico, este só visa a doença. Quando se fala em desinstitucionalização, se refere ao deslocamento da atenção institucional para a comunidade, sociedade. A reforma psiquiátrica surgiu justamente por isso, para que o portador de sofrimento psíquico não fosse visto como louco isoladamente, mas como ser humano capaz de exercer funções e interagir com o meio ${ }^{(15)}$.

O profissional de saúde pode se sentir inseguro no manejo do paciente psiquiátrico em crise, mesmo porque, nem sempre este se encontra capacitado para lidar com esse tipo de situação. Porém, para que a abordagem do paciente em crise seja realizado de maneira eficaz, é necessário que a equipe de enfermagem esteja capacitada para ficar exposta a todas as situações 
que possam ocorrer, nesse momento ${ }^{(16)}$. Isso porque, em cada crise o paciente reage de maneira diferente, embora os sinais e sintomas sejam peculiares. Desse modo, é preciso que o profissional de enfermagem tenha conhecimento e pensamento reflexivo para manter o equilíbrio psicológico e restabelecer o usuário para um quadro estável.

Diante de um quadro de crise, sentimentos como medo, insegurança e inutilidades foram explicitados pelos sujeitos do estudo. Baseado nesse contexto, cabe salientar sobre a necessidade de a equipe de enfermagem ter acompanhamento psicológico. É fundamental que os profissionais envolvidos saibam controlar suas emoções, para que estas não interfiram na intervenção prestada. Nesse sentido, para que a equipe de enfermagem tenha um bom desempenho com o paciente em crise, faz-se necessário que os profissionais reflitam e compreendam seus sentimentos e emoções, para poder direcionar sua atenção para a situação, e não perder o foco ${ }^{(16)}$.

Assim, parte-se do entendimento que o profissional capacitado possui mais habilidades e conhecimentos para lidar com o paciente em crise, do que aqueles que não receberam capacitação e/ou treinamento. Os profissionais treinados possuem técnicas e habilidades para cuidarem dos usuários com segurança.

É fundamental que os profissionais ofereçam um atendimento ao paciente com sofrimento psíquico em crise em sua totalidade e, para isso, é necessário respaldar-se em habilidades e conhecimentos específicos, e isso só é possível através da educação permanente desse profissional. Os treinamentos e/ou capacitações contribuem para que o profissional de enfermagem coloque o paciente em crise no centro, e dessa forma, se possa planejar o cuidado oferecido ${ }^{(2)}$.

\section{CONCLUSÕES}

Com base nos resultados, pode-se perceber que as práticas de enfermagem estão baseadas no novo modelo de cuidado dispensado na saúde mental, cujo objetivo é reduzir o número de internações psiquiátricas, promovendo atividades que resgatem a pessoa em situação de sofrimento psíquico para o espaço social. O estudo revelou pontos importantes; os profissionais de enfermagem têm facilidade para prestar atendimento ao paciente em situação de sofrimento psíquico, porém não se sentem totalmente capacitados e habilitados para esta situação.

Observou-se nas falas dos depoentes que, no momento de crise psiquiátrica, eles tentam acalmar o usuário dando espaço para a conversa e escuta.
Esta estratégia vem se destacando na reabilitação dos usuários porém, quando é necessário, são utilizados psicofármacos e os usuários são encaminhados para emergência psiquiátrica ou hospital psiquiátrico. Evidenciou-se também, que fatores intrafamiliares, uso incorreto da medicação e a abstinência, podem desencadear as crises psiquiátricas nos usuários em tratamento nos CAPS.

Frente ao exposto, percebeu-se que embora o estudo tenha revelado que os profissionais de enfermagem ainda apresentem fragilidades em lidar com o paciente em crise psiquiátrica, tal fato não os impede de prestar um cuidado qualificado e humanizado ao paciente com sofrimento psíquico, visando sua autonomia e ressocialização.

\section{REFERÊNCIAS}

1. Brusamarello T, Guimarães AN, Paes MR, Borba LO, Borille DC, Maftum MA. Cuidado de enfermagem em saúde mental ao paciente internado em hospital psiquiátrico. Cogitare enferm. 2009;14(1):79-84.

2. Almeida Filho AJ, Moraes AEC, Peres MAA. Atuação do enfermeiro nos centros de atenção psicossocial: implicações históricas da enfermagem psiquiátrica. Rev Rene. 2009;10(2):158-65.

3. Vietta EP, Kodato S, Furlan R. Reflexões sobre a transição paradigmática em saúde mental. Rev. LatinoAm. enfermagem. 2001;9(2):97-103.

4. Rocha RM. O Ser Humano, a saúde mental e os transtornos mentais de comportamento. In: Rocha RM. Enfermagem em Saúde Mental. 2a ed. Rio de Janeiro: Senac Nacional, 2010. p. 88-90.

5. Carvalho NR, Costa II. Primeiras Crises Psicóticas: identificação de pródromos por pacientes e familiares. Psic. clin. Rio de Janeiro. 2008;16(1):153-64.

6. Towsend MC. Enfermagem psiquiátrica conceitos de cuidados. $3^{\mathrm{a}}$ ed. Rio de Janeiro: Guanabara Koogan; 2002.

7. Tavares CMM. A educação permanente da equipe de enfermagem para o cuidado nos serviços de saúde mental. Texto Contexto Enferm. 2006;15(2):287-95.

8. Bardin L. Análise de conteúdo. $2^{\mathrm{a}}$ ed. Lisboa: Edições 70, 2009.

9. Pontes AC, Leitão IMTA, Ramos IC. Comunicação terapêutica em enfermagem: instrumento essencial 
do cuidado. Rev. bras. enferm. [Internet]. 2008;61(3) [acesso em 30 jun 2012]. Disponível: http://www.scielo. br/pdf/reben/v61n3/a06v61n3.pdf

10. Cardoso L, Galera SAF. Doentes mentais e seu perfil de adesão ao tratamento psicofarmacológico. Rev. Esc. Enferm. USP. 2009;43(1):161-7.

11. Borba LO, Paes MR, Guimarães AN, Labronici LM, Maftum MA. A família e o portador de transtono mental: dinâmica e sua relação familiar. Rev. Esc. Enferm. USP. 2011;42(5):442-9.

12. Malbergier A, Oliveira Jr HP. Dependência de tabaco e comorbidade psiquiátrica. Psicol. clin. 2005;32(5):27682.

13. Ministério da Saúde (BR). Secretaria de Atenção à Saúde. Saúde Mental no SUS: acesso ao tratamento e mudança do modelo de atenção: Relatório de Gestão 2003-2006. Brasília; 2007.

14. Campos CJG, Teixeira MB, O atendimento do doente mental em pronto-socorro geral: sentimentos e ações dos membros da equipe de enfermagem. Rev. Esc. Enferm. USP. 2001;35(2):141-9.

15. Gonçalves AM, Sena RR. A reforma psiquiátrica no Brasil: contextualização e reflexos sobre o cuidado com o doente mental na família. Rev. Latino-Am. enferm. 2001;9(2):48-55

16. Stuart GW, Laraia MT. Enfermagem psiquiátrica: princípios e prática. Porto Alegre: Artmed; 2001. 